\title{
Low-resolution spectroscopy and spectral energy distributions of selected sources towards $\sigma$ Orionis
}

\author{
J. A. Caballero ${ }^{1}$, L. Valdivielso ${ }^{2}$, E. L. Martín ${ }^{2,3}$, D. Montes ${ }^{1}$, S. Pascual ${ }^{1}$, and P. G. Pérez-González ${ }^{1}$ \\ 1 Dpto. de Astrofísica y Ciencias de la Atmósfera, Facultad de Física, Universidad Complutense de Madrid, 28040 Madrid, Spain \\ e-mail: caballero@astrax.fis.ucm.es \\ 2 Instituto de Astrofísica de Canarias, Avenida Vía Láctea, 38200 La Laguna, Tenerife, Islas Canarias, Spain \\ 3 University of Central Florida, Dept. of Physics, PO Box 162385, Orlando, FL 32816-2385, USA
}

Received 15 July 2008 / Accepted 2 September 2008

\section{ABSTRACT}

\begin{abstract}
Aims. We study in detail nine sources in the direction of the young $\sigma$ Orionis cluster, which is considered to be a unique site for studying stellar and substellar formation. The nine sources were selected because of their peculiar properties, such as extremely-red infrared colours or excessively strong $\mathrm{H} \alpha$ emission for their blue optical colours.

Methods. We acquired high-quality, low-resolution spectroscopy $(R \sim 500)$ of the nine targets with ALFOSC at the Nordic Optical Telescope. We also re-analysed [24]-band photometry from MIPS/Spitzer and compiled the highest quality photometric dataset available at the $V i J H K_{\mathrm{s}}$ passbands and the four IRAC/Spitzer channels, for constructing accurate spectral energy distributions between 0.55 and $24 \mu \mathrm{m}$.

Results. The nine targets were classified into: one Herbig Ae/Be star with a scattering edge-on disc; two G-type stars; one X-ray flaring, early-M, young star with chromospheric $\mathrm{H} \alpha$ emission; one very low-mass, accreting, young spectroscopic binary; two young objects at the brown-dwarf boundary with the characteristics of classical T Tauri stars; and two emission-line galaxies, one undergoing star formation, and another whose spectral energy distribution is dominated by an active galactic nucleus. We also discovered three infrared sources associated with overdensities in a cold cloud of the cluster centre.

Conclusions. Low-resolution spectroscopy and spectral energy distributions are a vital tool for measuring the physical properties and evolution of young stars and candidates in the $\sigma$ Orionis cluster.
\end{abstract}

Key words. stars: emission-line, Be - stars: low-mass, brown dwarfs - stars: pre-main sequence -

Galaxy: open clusters and associations: individual: $\sigma$ Orionis - galaxies: quasars: emission lines

\section{Introduction}

The $\sigma$ Orionis cluster is one of the few star-forming regions within $1 \mathrm{kpc}$ that contain massive stars (B2 and earlier - Zinnecker \& Yorke 2007). In particular, the Trapezium-like system $\sigma$ Ori, which provides its name to the cluster, contains five known stars with masses above $\sim 7 M_{\odot}(\mathrm{A}, \mathrm{B}, \mathrm{D}, \mathrm{E}$, and the recently confirmed $\mathrm{F}$ component - Caballero 2007a, and references therein; Peterson et al., in prep.). The nearby Horsehead Nebula, whose mane is illuminated by the Trapezium-like system, acts as a retaining wall to the east, while approximately $80 \%$ of the cluster members are contained in a circular pool of only 20 arcmin radius, where extinction is low (Lee 1968; Béjar et al. 2004; Caballero 2008a). Although uncertainties remain regarding its true heliocentric distance $(d \sim 350-440 \mathrm{pc}-$ Caballero 2008b; Mayne \& Naylor 2008; Sherry et al. 2008), $\sigma$ Orionis was classified as the closest massive-star forming region (Zinnecker \& Yorke 2007). Furthermore, with $\sim 3 \mathrm{Ma}$ (Zapatero Osorio et al. 2002a; Oliveira et al. 2006; Caballero 2008b), the cluster has an age intermediate between those of extremely young, highly embedded ( $\sim 1 \mathrm{Ma}$ : Orion Nebula Cluster, NGC 2024$)$ and relatively clear, yet very young clusters ( 5$10 \mathrm{Ma}: \lambda$ Orionis, NGC 2023) at similar heliocentric distances $(d \sim 400 \mathrm{pc})$.

Its low absorption, relative closeness, and youth imply that $\sigma$ Orionis is one of the most fruitful hunting grounds for substellar objects (Béjar et al. 1999, 2001, 2004; Scholz \& Eislöffel 2004; Kenyon et al. 2005). In particular, the cluster accounts for more than a half of all known confirmed, isolated, planetary-mass objects in the literature (Zapatero Osorio et al. 2000, 2002b,c, 2007; Barrado y Navascués et al. 2001; Martín et al. 2001a; González-García et al. 2006; Caballero et al. 2007; Scholz \& Jayawardhana 2008; Luhman et al. 2008). In Caballero (2008c) the reader can find additional cluster data and useful references for all subjects that have been completed for $\sigma$ Orionis (from X-ray emission, through Herbig-Haro objects, to brown dwarfs with discs).

To obatin a deeper understanding of the processes that occur in the cluster, Caballero (2008c) tabulated, in his Mayrit catalogue, $241 \sigma$ Orionis stars and brown dwarfs with known feaures of youth (e.g. OB spectral types, Li I $\lambda 6707.8 \AA$ in absorption, abnormal strength of other alkali lines because of low surface gravity, $\mathrm{H} \alpha \lambda 6562.8 \AA$ in strong emission due to accretion, infrared flux excess due to surrounding disc), 97 candidate cluster members, and 115 back- and foreground sources. Of the 338 cluster members and member candidates, 54 are fainter than the stellar/substellar boundary at $J \approx 14.5 \mathrm{mag}$ for null extinction (Caballero et al. 2007). The Mayrit catalogue is to date the most comprehensive database of $\sigma$ Orionis members, and is being used as input to investigating the cluster spatial distribution, the frequency of discs at different mass intervals, the X-ray emission, the occurrence of wide binaries, or the initial mass function 
from about $18 M_{\odot}$ to a few Jupiter masses (Caballero 2008b; Luhman et al. 2008; López-Santiago \& Caballero 2008). Any systematic error in the catalogue (e.g. incompleteness, contamination) may have a negative influence on the previous results. It is therefore necessary to confirm or discard membership in the $\sigma$ Orionis cluster of many Mayrit sources for which we do not have spectroscopic data. Some disputed sources were observed by Maxted et al. (2008), Gatti et al. (2008), and Sacco et al. (2008). However, dozens of cluster members and member candidates remain without spectroscopy.

\section{Observations and analysis}

\subsection{The target sample}

We present low-resolution spectroscopy and spectral energy distributions (from 0.55 to $24 \mu \mathrm{m}$ ) of nine selected sources towards the $\sigma$ Orionis cluster. We selected these nine targets based on a variety of properties derived from existing catalogues (peculiar $\mathrm{H} \alpha$ emission, possible extended point spread function, abnormally-red infrared colours, X-ray flares). The earliest and latest expected spectral types were B to late M, covering a magnitude interval of $\Delta V \approx 9$ mag (which represents a factor $\sim 4000$ in flux, or a factor $\sim 30$ in mass). We provide in Table 1 the names, coordinates, and references of the nine targets. Falsecolour composite images centred on each target, with different wavelength combinations, are available upon request from the authors.

\subsection{Low-resolution optical spectroscopy with ALFOSC}

We used the Andalucía Faint Object Spectrograph and Camera (ALFOSC) at the $2.56 \mathrm{~m}$ Nordic Optical Telescope (NOT) in the Spanish Observatorio del Roque de Los Muchachos during five nights in Oct. 2006. The ALFOSC detector was the CCD\#8, a nimo, back-illuminated E2V $2 \mathrm{k} \times 2 \mathrm{k} 42-40$ with a plate scale of 0.19 arcsec/pixel $(2 \times 2$ binning $)$. With the grism \#5 and the 1.0 arcsec-size slit, we achieved a dispersion of $3.1 \AA /$ pixel and a resolution of $14.2 \AA$ at $7000 \AA(R \equiv \lambda / \Delta \lambda=494)$.

The total wavelength coverage was $5350-9910 \AA$. The central wavelength $(7630 \AA)$ was red-shifted with respect to the effective blaze wavelength $(6500 \AA)$ to maximise the use of the reddest part of the optical spectrum, where late $\mathrm{M}$ stars (our faintest objects) emit most of their energy. With the grism \#5, the second order (corresponding to bandpasses $B$ and $V$ ) is noticeable at the $20 \%$ level only at wavelengths $\lambda>9700 \AA$ (no blocking filter was therefore used). Unfortunately, the peak-topeak fringe levels start at $6700 \AA$ and increase in intensity redwards ( $8 \%$ at $7500 \AA, 18 \%$ at $8000 \AA,>30 \%$ at $>9000 \AA)$. The final useful wavelength coverage was 5500-9300 $\AA$ due to both fringing and a low number of counts in flux standard star spectra at the edges of the wavelength range, caused by the lower quantum efficiency of the detector.

The weather during our run was, in general, fair with high, thin cirruses only during the final night. The seeing varied in the range $\sim 0.7-1.0$ arcsec, which justified our slit-size choice. Wind speed was low and humidity remained approximately stable during each night (with maximum variations of 15\%). The dates of observation and the exposure times of the nine (combined) spectra are provided in Table 1. Total exposure times ranged from only $30 \mathrm{~s}$ for the brightest target to $1 \mathrm{~h} 20 \mathrm{~min}$ for the faintest cluster member. Bias images for posterior calibration were taken at sunsets, while flats and arcs were obtained just before and after each scientific spectrum. The spectra of the five faintest targets are the combination of between two to four individual spectra, sometimes taken on different nights (e.g. the final spectrum of Mayrit 264077 is the combination of two 900 s-long spectra taken on 2006 Oct. 11 and 12). We also acquired spectra of GJ 3517 (M9V) during nighttime. This object was used as a flux, spectral-type, and radial-velocity standard/comparison star.

Reduction of the raw images (bias subtraction and flatfielding), spectrum extraction, flux calibration, and combination of the spectra (in this order), was carried out using standard procedures within the IRAF environment (daophot.onedspec). We applied a tiny Doppler shift of $2 \AA$ to the spectra taken during the first two nights due to a systematic error in the calibration. Since the resolution power was $14.2 \AA$ (measured from the arcs and sky lines), this shift had no implications on further analysis steps. Before combination, we checked that there were no significant differences between individual spectra (e.g. no appreciable variation in the $\mathrm{H} \alpha$ emission).

The nine final (combined) spectra are shown in Figs. 2 and 3. We note the strong telluric absorption features, especially the $\mathrm{O}_{2}$ A and B bands at 6850-6960 and 7550-7710 and another patent water-vapour absorption feature at $\lambda=7150$ $7340 \AA$ (there is other strong feature at $>9360 \AA$, not visible in our figure).

\subsection{Multi-wavelength photometry}

\subsection{1. $\mathrm{V} \mathrm{i} \mathrm{J} \mathrm{H} \mathrm{K} \mathrm{s}_{\mathrm{s}}$ [3.6] [4.5] [5.8] [8.0]-band photometry}

We collected optical, red optical, near-infrared, and mid-infrared magnitudes from the literature for the nine sources. The $i$ and $J H K_{\mathrm{s}}$ magnitudes were retrieved homogeneously from the Deep Near Infrared Survey of the Southern Sky (DENIS, Epchtein et al. 1997) and the Two Micron All Sky Survey (2MASS, Skrutskie et al. 2006). The magnitudes in the four channels of the Infrared Array Camera (IRAC, 3.8-8.0 $\mu \mathrm{m}$ ) on the Spitzer Space Telescope were taken either from Caballero et al. (2007, 2008) or from Hernández et al. (2007), depending on availability. Finally, we compiled the most accurate available visual magnitudes for each target from a heterogeneous collection of sources. We provide in Table 2 all necessary information, including the magnitudes and their origin.

\subsection{2. [24]-band photometry with MIPS/Spitzer and spectral energy distributions}

Hernández et al. (2007) tabulated $24 \mu \mathrm{m}$ Multiband Imaging Photometer for Spitzer (MIPS) magnitudes ([24]) for a few of our targets. To expand the wavelength coverage of our spectral energy distributions, we downloaded with the Leopard tool the most recent post-Basic Calibrated Data [24]-band MIPS mosaic covering the entire $\sigma$ Orionis cluster (P.I.: G. Rieke, programme: Evolution and Lifetimes of Protoplanetary Disks).

First, we searched for sources suitable for study of their observed [24]-band flux. As can be seen in Caballero et al. (2008) and Hernández et al. (2007), intracluster clouds (some associated with the Horsehead Nebula), hardly visible in both the optical and near-infrared, are apparent at wavelengths redwards of $\sim 8 \mu \mathrm{m}$ (see Appendix A). To avoid contamination by spurious detections, we imposed a relatively high threshold on the source finding at $10 \sigma$ over the noise background. We next performed standard aperture and point-spread-function (PSF) photometry on the mosaic with the IRAF package digiphot, using an aperture radius of 13 arcsec and a background annulus of between 39 


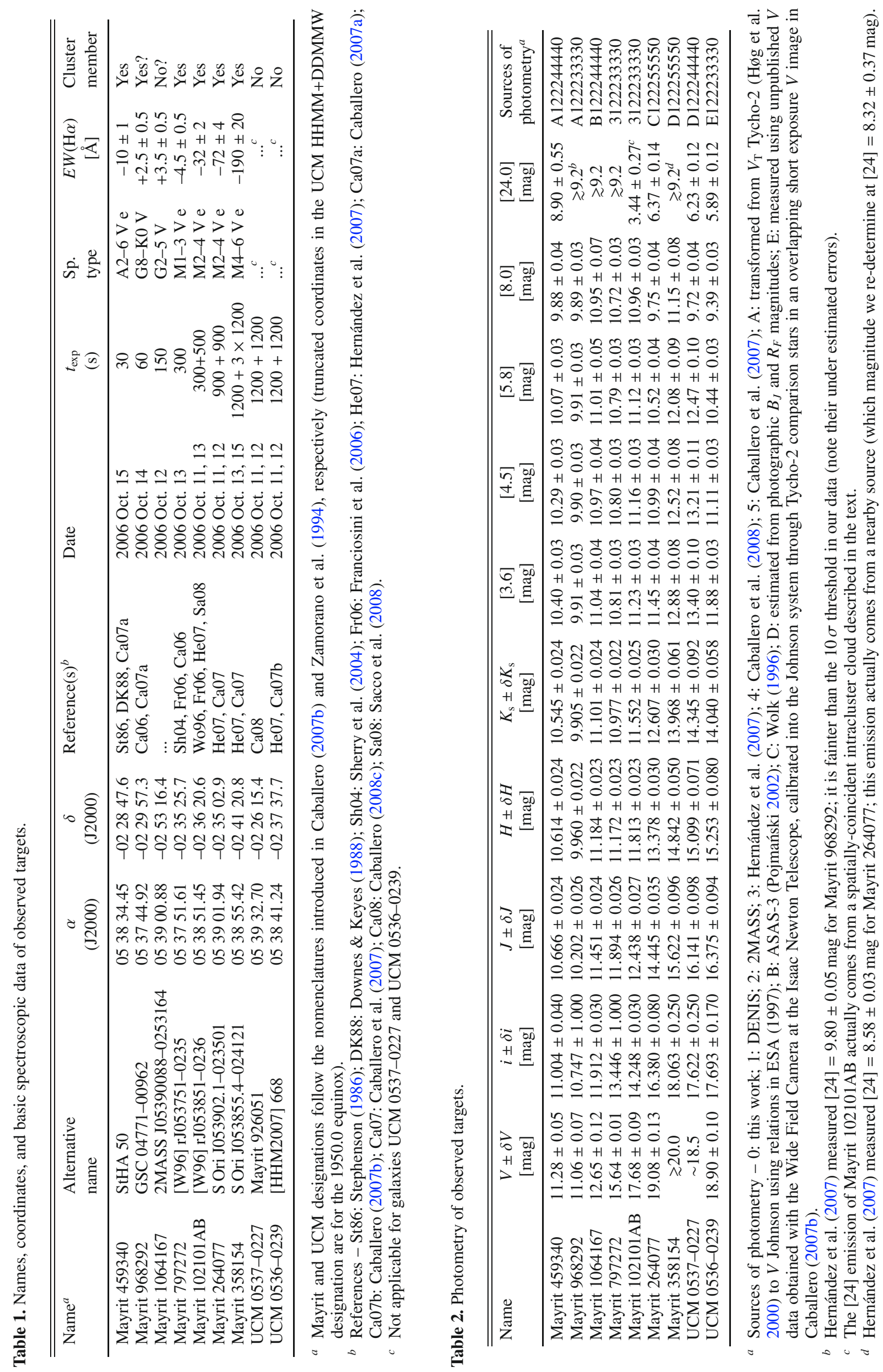



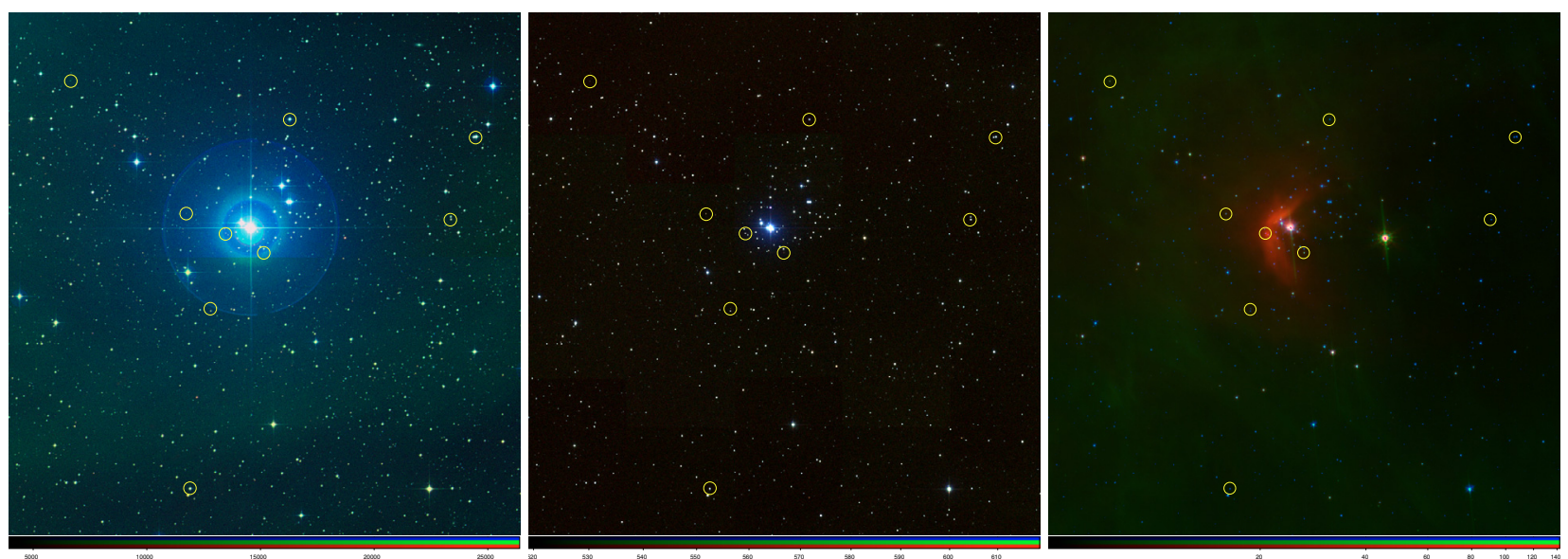

Fig. 1. False-colour composite images centred approximately on $\sigma$ Ori at different wavelengths. From left to right: optical photographic $B_{J}+R_{F}+I_{N}$, 2MASS $J+H+K_{\mathrm{s}}$, and Spitzer [3.6] + [8.0] + [24] (blue + green + red). Approximate size is $30 \times 30 \mathrm{arcmin}^{2}$; north is up, east is left. The nine analysed targets are encircled. We note the relative dimming of the Trapezium-like star system and the appearence of a large dust cloud eastwards at the reddest wavelengths (see Sect. A). Colour version of all our figures are available in the electronic publication.

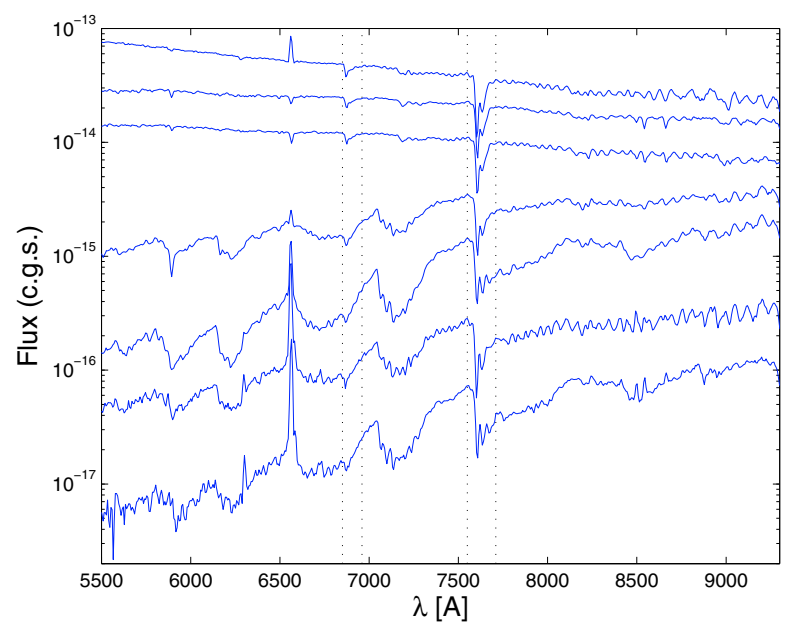

Fig. 2. Flux-calibrated, low-resolution, ALFOSC/NOT, spectra of the stars and brown dwarfs Mayrit 459340, Mayrit 968292, Mayrit 1064167, Mayrit 797272, Mayrit 102101AB, Mayrit 264077, and Mayrit 358154, from top to bottom. Vertical dotted lines indicate strong, telluric-absorption features. Beyond $\sim 8000 \AA$, ripples indicate regions where fringing contributes to the spectra.

and 48 arcsec (which corresponded to an aperture correction of 1.175 according to the Spitzer Science Centre ${ }^{1}$ ). We used $F_{\nu 0}=$ $7.17 \pm 0.11 \mathrm{Jy}$ and $\lambda_{0}=23.675 \mu \mathrm{m}$ for transforming PSF fluxes into apparent magnitudes. In the previous-to-the-last column in Table 2, we provide the measured [24] magnitudes and their errors, computed from the quadratic sum of the error in the PSF photometry and the errors in both the aperture correction and absolute calibration ( 7\%, Engelbracht et al. 2007). The $10 \sigma$ threshold implied that the completeness limit of our [24]-band survey was $\sim 9.2 \mathrm{mag}$.

Magnitudes for only five of our nine main targets were derived. The tabulated magnitude of one target (Mayrit 102101AB) corresponds to a bright knot in a cloud in the north-east vicinity of $\sigma$ Ori, which is spatially-coincident with our star (see again Sect. A). The other four stars without MIPS measurements have, therefore, magnitudes [24] $\gtrsim 9.2 \mathrm{mag}$.

${ }^{1}$ http://ssc.spitzer.caltech.edu/mips/apercorr/
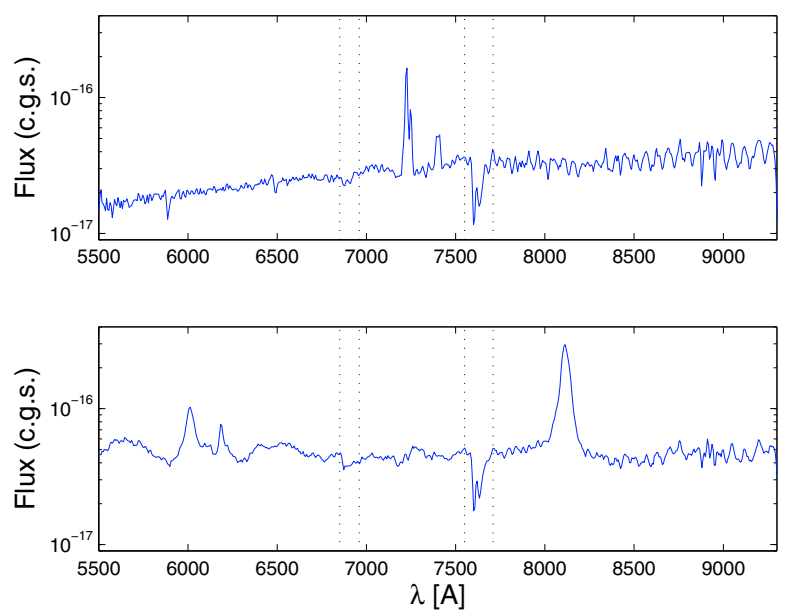

Fig. 3. Same as Fig. 2, but for the emission-line galaxies UCM 05370227 (top window) and UCM 0536-0239 (bottom window).

Using the magnitudes in Table 2 and the zero-point values for conversion between magnitudes and flux densities compiled in the NASA/IPAC/MSC Star and Exoplanet Database ${ }^{2}$, we constructed the spectral energy distributions shown in Fig. 4.

\section{Results and discussion}

We classified our nine targets into three categories, depending on their astrophysical nature. The classes were early-type and solar-like stars, late-type young stars and brown dwarfs, and emission-line galaxies at intermediate redshifts. In the rightmost columns of Table 1, we list the spectral types, equivalent widths of $\mathrm{H} \alpha$, and cluster membership measured for seven young object candidates. Actually, we measured the pseudo-equivalent width $(p E W)$ of the lines with respect to the pseudo-continuum of the four M-type stars and brown dwarfs. This definition extends to other lines different from the Balmer series (e.g. Li I). Hereafter, we use the term equivalent width $(E W)$ for simplicity. The error in the $E W$ s originates in the uncertainty in the determination of the (pseudo-)continuum.

\footnotetext{
${ }^{2}$ http://nsted.ipac.caltech.edu/NStED/docs/parhelp/ Photometry.html.
} 

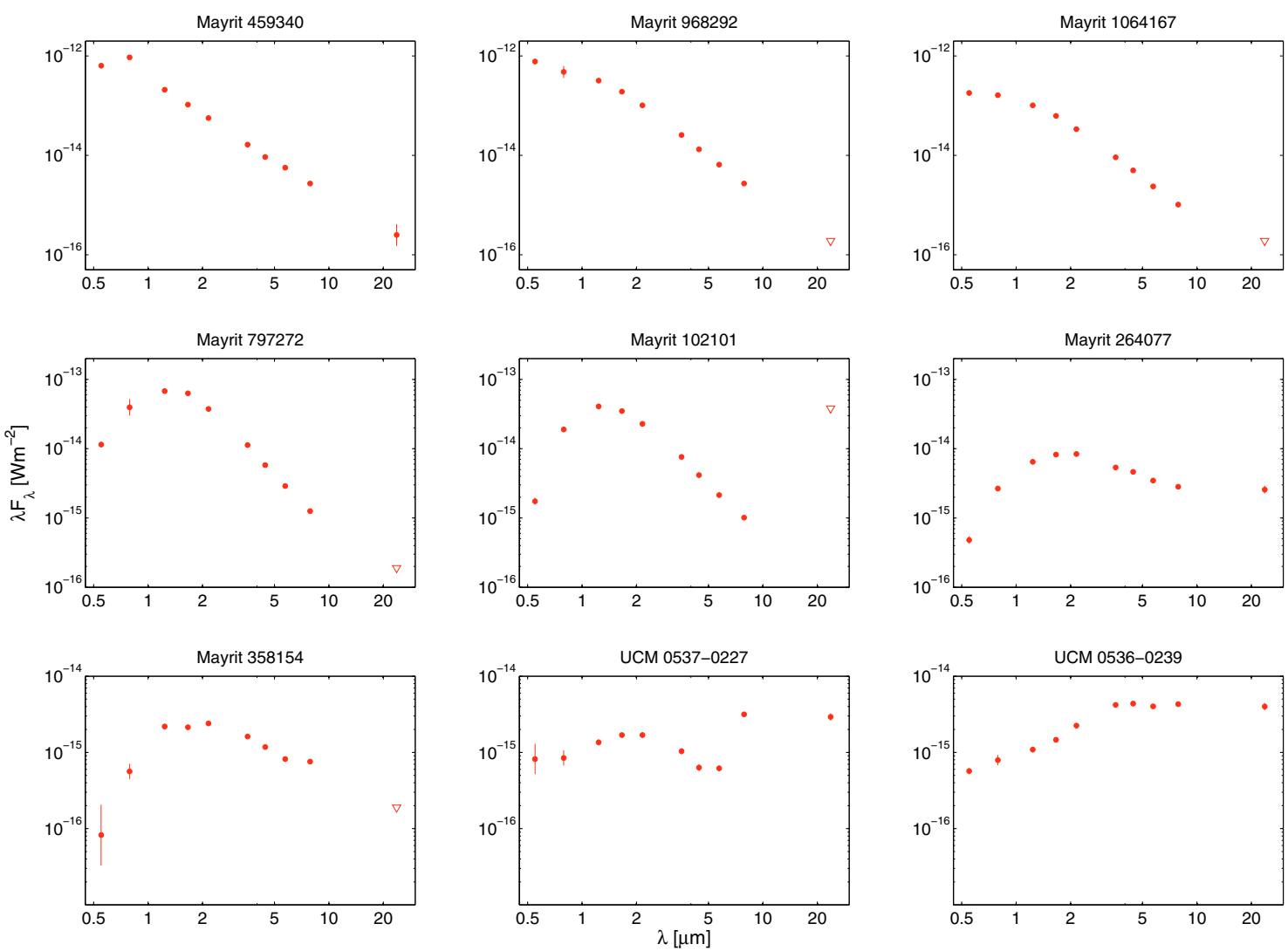

Fig. 4. Spectral energy distributions of the nine targets in Tables 1 and 2. From the shortest to the longest wavelengths, the data points correspond to the passbands Johnson $V$, DENIS $i$, 2MASS $J, H$, and $K_{\mathrm{s}}$, IRAC/Spitzer [3.6], [4.5], [5.8], and [8.0], and MIPS/Spitzer [24]. The lower triangle indicates an upper limit for the flux at the [24] passband.

The seven young object candidates and the two galaxies are discussed in detail in the following sections.

\subsection{Early-type and solar-like stars}

\subsubsection{Mayrit 459340}

Mayrit 459340 (StHA 50) is an $\mathrm{H} \alpha$ emission star found above 10 deg Galactic latitude by Stephenson (1986), who investigated a large number of red-sensitive objective prism plates. Soon afterwards, Downes \& Keyes (1988) classified Mayrit 459340 as a Be star from a spectrum taken with an image-tube scanner. It went unstudied until Caballero (2007a) listed it as one of the (30) brightest stars in the $\sigma$ Orionis cluster (Mayrit 459340, with $M \gtrsim$ $1.4 M_{\odot}$, is also one of the most massive cluster stars). According to Caballero (2007a), Mayrit 459340 deviates bluewards of the spectro-photometric cluster sequence in a $V$ vs. $B-V$ colourmagnitude diagram from Tycho-2 magnitudes at the level of $3 \sigma$. Despite its early $(\mathrm{Be})$ spectral-type matches, within uncertainties, its blue colours in the optical $\left(B_{\mathrm{T}}-V_{\mathrm{T}}=-0.06 \pm 0.11 \mathrm{mag}\right)$, Mayrit 459340 is $\Delta B_{\mathrm{T}}=1.96 \pm 0.06 \mathrm{mag}$ fainter than the faintest B-type star in $\sigma$ Orionis (HD 294275 Mayrit 1227243, B9V, Caballero 2007a). Its $V_{\mathrm{T}}$ magnitude is in closer agreement with that of an early- or mid-F spectral-type star in the cluster, or a late-B spectral-type star at an unlikely heliocentric distance a factor of three higher than to $\sigma$ Orionis. The first hypothesis would easily explain its $\mathrm{H} \alpha$ emission, while the second hypothesis would locate Mayrit 459340 at about $300 \mathrm{pc}$ below the Galactic plane (the Galactic vertical scale height of OBA-type stars is only about 100 pc; e.g. Bahcall \& Soneira 1980).
Caballero (2007a) also reported that Mayrit 459340 has a disc based on 3.6-8.0 $\mu \mathrm{m}$ IRAC/Spitzer photometry from Caballero et al. (2008). Our MIPS/Spitzer [24] photometry (tabulated in Table 2) corroborated his statement: we derived colours $[3.6]-[24]=1.5 \pm 0.6 \mathrm{mag}$ and [3.6] $-[8.0]=0.52 \pm 0.05 \mathrm{mag}$, that deviate by about $3 \sigma$ and $10 \sigma$ from the expected values for a disc-less dwarf ([3.6] - [24] [3.6] - [8.0] 0 mag). From the ALFOSC spectrum, shown in the top of Fig. 2, we measured an intense $\mathrm{H} \alpha$ emission of $-10 \pm 1 \AA$ and derived a spectral type in the interval A2-6V, which corresponds to a most probable mass in the interval $3-4 M_{\odot}$. This spectral type agrees approximately with the blue $B_{\mathrm{T}}-V_{\mathrm{T}}$ colour of Mayrit 459340 and a previous determination by Downes \& Keyes (1988). In deciding the classification, we investigated the depth of the blended doublets He I D $\lambda \lambda 5875.6,5876.0 \AA$ and $\mathrm{Na} \mathrm{I} \lambda \lambda 5890.0,5895.6 \AA$ and the line Ca I $25857.5 \AA$, and compared the continuum slope in its spectrum with those of early-type standard stars from Valdes et al. (2004). The strong $\mathrm{H} \alpha$ emission appears to persist with time, from 1978-1984 (Stephenson 1986), through 1986-1987 (Downes \& Keyes 1988), to the present (this work). The absorption feature of the diffuse interstellar band at about $6280 \AA$, typical of early-type stars in young clusters, is also quite clear. To our knowledge, there is only one scenario that can explain simultaneously the A spectral type, the $\mathrm{H} \alpha$ emission, the abnormal blue colour for its magnitude (or, alternatively, its abnormal faintness for its colour), and the flux excess at $8-24 \mu \mathrm{m}$ : the blueing effect in a Herbig $\mathrm{Ae} / \mathrm{Be}(\mathrm{HAeBe})$ star with a scattering edge-on disc (Thé et al. 1994, and references therein).

There are grounds for considering Mayrit 459340 a (longscale) photometric variable, which is another characteristic typical of HAeBe stars. From data of the All Sky Automated Survey 
(ASAS-3, Pojmański 2002), we measured $\bar{V}=11.312 \mathrm{mag}$, $\sigma_{V}=0.045 \mathrm{mag}$, and $\overline{\delta V}=0.032 \mathrm{mag}(N=248)$, consistent with both the Tycho- $2 V_{\mathrm{T}}$ measurement and with the lack of large-amplitude photometric variations $(\Delta V \gg 0.045 \mathrm{mag})$ in Mayrit 459340 during the years from 2000 to 2005 . There is no evidence of photometric variability either on the basis of the data available from the USNO-B1.0 catalogue (Monet et al. 2003). However, from the spectral energy distribution, the DENIS $i$ magnitude, measured in $1996 \mathrm{Feb} .2$, appears to be $\sim 0.6-0.7 \mathrm{mag}$ brighter than expected. The value $i=11.00 \pm 0.04 \mathrm{mag}$ is still far away from the $i \sim 10.0$ mag threshold below which brighter DENIS stars in $\sigma$ Orionis are affected by saturation and non-linear effects (Caballero 2008c). From the ratio between our flux-calibrated spectra, we also estimated that during the observations (2006 Oct.), Mayrit 459340 was $\sim 0.9 \mathrm{mag}$ brighter at $6200-6300 \AA$ than the solar-like, young, star candidate Mayrit 968292. However, from the compiled magnitudes in Table 2, Mayrit 459340 is brighter than Mayrit 968292 only for $\lambda \gtrsim 8 \mu \mathrm{m}$ (where the flux excess due to the disc becomes more important). Furthermore, the tabulated $V_{\mathrm{T}}$ magnitudes were measured in the same epoch (early 1990s), and Mayrit 459340 was $0.17 \pm 0.12 \mathrm{mag}$ fainter than Mayrit 968292 at the corresponding effective wavelength of $5320 \AA$. This apparent brightening at recent epochs, if accompanied by an appropiate colour change, would imply that the data for Mayrit 459340 are located on the right spectro-photometric sequence of $\sigma$ Orionis. Recalling the scattering edge-on disc scenario of Thé et al. (1994), "the variations in brightness and in colour [of a HAeBe star] are caused by a dust cloud in a Keplerian orbit revolving in the outer part of a star's circumstellar disc". Most of the time, the disc of Mayrit 459340 is orientated almost edge-on with respect to the observer, and the star is well obscured by the dust cloud. It is at this stage that the dimming is at its maximum, and scattered blue light is more easily detected. It is expected that when the dust cloud passes away from the front of the HAeBe star, it will become brighter and redder. This effect has been clearly detected in the A3e-type, variable star (with rapid variations) UX Ori (Bibo \& Thé 1991). Mayrit 459340 is about 2.6 mag fainter than UX Ori, which is probably why any variations have remained unnoticed until now. High-resolution spectroscopy would enable more robust constraints of membership by means of measurements of radial velocity.

\subsubsection{Mayrit 968292 and Mayrit 1064167}

On the one hand, the Tycho-2 star Mayrit 968292 (GSC 0477100962) was the brightest (in the optical) $\sigma$ Orionis member candidate in the Mayrit catalogue without spectroscopy (Caballero 2008c). This was a per se reason for first acquiring an optical spectrum with ALFOSC. At $V \approx 11.1 \mathrm{mag}$, an exposure time of only 1 min was required. Mayrit 968292 is the brightest component of a visual binary with a relatively small angular separation $(\rho \approx 9.8 \operatorname{arcsec}, \theta \approx 97 \mathrm{deg}, \Delta J=0.72 \pm 0.03 \mathrm{mag})$. The secondary, Mayrit 958292, displayed an apparent lithium absorption feature in a spectrum taken by Caballero (2006), which confirmed the star to be a very young member of the Ori OB1b association. If both stars formed a physical pair, then it would be one of the few wide binaries $(r \approx 3800 \mathrm{AU})$ detected so far in the $\sigma$ Orionis cluster (several wide binary candidates were listed in Caballero 2007b). See also Caballero (2007a) for a consideration on the possible X-ray emission of Mayrit 968292.

Mayrit 1064167 (2MASS J05390088-0253164) was, however, an unknown star, whose position in the $i$ versus $i-K_{\mathrm{S}}$ diagram implied that it was a candidate fore- and background star, but still close to the sequence of the cluster (see Fig. 4 of Caballero 2008c). It is the brightest of a trio of stars separated by $20-40$ arcsec. One of the other two components of the asterism also has optical colours and magnitudes that are typical of solar-like cluster members (e.g. Sherry et al. 2004). However, Caballero (2006) found no trace of lithium in a high signal-to-noise, mid-resolution spectrum of the target. The remaining star in the trio is a foreground late-type dwarf or giant based on a relatively large proper motion and quite red optical/near-infrared colours for its magnitude $\left(J-K_{\mathrm{s}}=\right.$ $1.30 \pm 0.04 \mathrm{mag}$, Caballero 2008c). Instead of extrapolating the non-membership also to Mayrit 1064167, we found three X-ray events tabulated by the Second ROSAT Position-Sensitive Proportional Counter (PSPC) Catalogue, 2RXP (ROSAT 2000), and the White-Giommi-Angelini version of the ROSAT PSPC Catalogue, WGACAT (White et al. 2000), at 10-30 arcsec to the star. Although the X-ray events could also be associated with a background extragalactic source, we decided to test photometric selection criteria for cluster members in the literature (Sherry et al. 2004; Caballero 2008c) using the possible X-ray emitter Mayrit 1064167.

At a first glance of the ALFOSC spectra and spectral energy distributions of Mayrit 968292 and Mayrit 1064167, the two stars appeared to be G-type stars with no indication of accretion or infrared flux excess. We could only impose lower limits to the [24]-band magnitudes. A careful comparison of the blended $\mathrm{Na}$ I $\mathrm{D}_{1} \mathrm{D}_{2}$ doublets and $\mathrm{H} \alpha$ lines indicated that Mayrit 968292 is cooler than Mayrit 1064167 (the absorption of the Na I doublets and the $\mathrm{H} \alpha$ line increases and decreases, respectively, towards later spectral types). By comparison with several spectraltype standard stars taken from Jacoby et al. (1984), Montes et al. (1997), and Valdes et al. (2004), we classified Mayrit 968292 as G8-K0V and Mayrit 1064167 as G2-G5V. We obtained a similar classification using the lines ratio $\mathrm{H} \alpha / \mathrm{Fe}$ I $\lambda 6495.0 \AA$ (Danks \& Dennefeld 1994). Other lines typical of solar-like stars, observed in the spectra of Mayrit 968292 and Mayrit 1064167, were the calcium features Ca I $26163.8 \AA$ and, especially, the infrared triplet Ca II $\lambda \lambda \lambda 8498.0,8542.1,8662.1 \AA$ (partially embedded in the fringing region). Unfortunately, our spectra are not of sufficient spectral resolution to detect clearly any other spectroscopic diagnostic of youth (especially lithium).

Although Mayrit 1064167 has an earlier spectral type than Mayrit 968292, the first star is about $1.2 \mathrm{mag}$ fainter than the second at all passbands. Since such a large reversal of brightness is improbable, we consider that at least one of the two stars is a contaminant. The interloper is probably Mayrit 1064167, because Mayrit 968292 follows the cluster sequence and Mayrit 1064167 does not (it has roughly the same magnitudes as mid-K-type young stars in $\sigma$ Orionis). Albeit Mayrit 968292 remains a good solar-like, cluster-member candidate, it is necessary to obtain higher-resolution spectroscopy to confirm its membership. If its youth is verified, its membership in the Mayrit 968292-958292 binary will remain unknown for a longer time: because of the location of $\sigma$ Orionis in the solar antapex, its cluster members have very low proper motions $(\mu<$ 10 mas a $^{-1}$, Caballero 2007a).

\subsection{Late-type young stars and brown dwarfs}

We used primarily the PC3 index (Martín et al. 1996, 1999) for the spectral-type classification of the four M-type objects, although we also took into account the information provided by 
the I1, I2, and I3 indices (indicators of the strength of several $\mathrm{CaH}$ and $\mathrm{TiO}$ absorption bands, Martín \& Kun 1996) and some indices associated with metallic hydrides and oxides $(\mathrm{CrH}, \mathrm{FeH}$, $\mathrm{H}_{2} \mathrm{O}, \mathrm{TiO}$, and $\mathrm{VO}$ ). We also compared the index values with those of spectral standards in the literature and of GJ 3517, observed with the same instrumental configuration.

\subsubsection{Mayrit 797272}

Mayrit 797272 ([W96] rJ053751-0235) is a moderate X-rayemitting, early-M star in $\sigma$ Orionis. It was first discovered by Wolk (1996), who classified it as a ROSAT M0 star with lithium in absorption $(E W(\mathrm{Li} \mathrm{I})=+0.32 \AA)$ and $\mathrm{H} \alpha$ in faint chromospheric emission $(E W(\mathrm{H} \alpha)=-6.4 \AA)$. A decade later, Franciosini et al. (2006) measured its X-ray count rate with $X M M-N e w t o n$ and estimated an M2 spectral type from the $R-I$, $V-I$, and $I-J$ colours using the relations by Kenyon \& Hartmann (1995) and Leggett et al. (2001). Almost simultaneously, Caballero (2006) acquired a low signal-to-noise, optical spectrum in which the $\mathrm{H} \alpha$ line appeared to correspond to faint, broadened emission and the Li I line was undetected. López-Santiago \& Caballero (2008) found Mayrit 797272 to be an X-ray flaring star from new XMM-Newton data. Finally, Mayrit 797272 forms a visual binary $(\rho \approx 10.5 \operatorname{arcsec}, \theta \approx 5 \mathrm{deg}$ ) with a slightly brighter star that does not seem to belong to the $\sigma$ Orionis cluster.

We have derived the most probable spectral type of Mayrit 797272 in the range M1-3V from the ALFOSC spectrum shown in Fig. 2. Our determination is consistent with previous analyses. The $\mathrm{H} \alpha$ line in our spectrum of Mayrit 797272 is distinctive with an equivalent width similar to that measured by Wolk (1996). The broad emission detected by Caballero (2006) was possibly due to the low signal-to-noise ratio of the data and background contamination by a nearby fibre (he used a multifibre spectrograph). The chromospheric $\mathrm{H} \alpha$ emission and the absence of flux excess at $\lambda \lesssim 8 \mu \mathrm{m}$ suggest that Mayrit 797272 lacks a disc, which would lead to the star rotating more slowly. The X-ray variability is easier to explain for a young, fastrotating, disc-free, almost completely-convective object such as Mayrit 797272 (Güdel et al. 1995; Feigelson et al. 2002; Stelzer et al. 2004; Robrade \& Schmitt 2005).

\subsubsection{Mayrit $102101 \mathrm{AB}$}

Mayrit 102101AB ([W96] rJ053851-0236) was, like Mayrit 797272, first detected as a ROSAT X-ray source by Wolk (1996). He derived a K5 spectral type and measured $E W(\mathrm{H} \alpha) \approx-86 \AA$ in a faint spectrum in which he was unable to detect the $\mathrm{Li}$ I line. The star was later detected with XMM-Newton and Chandra by Franciosini et al. (2006), Caballero (2007b), and Skinner et al. (2008). Using IRAC and MIPS/Spitzer data, Hernández et al. (2007) claimed that Mayrit 102101AB harbours an "evolved disc" (i.e. the star exhibits smaller IRAC excesses than optically thick disc systems). Remarkably, Mayrit 102101AB is one of the few low-mass, spectroscopic binaries (SB2) in the $\sigma$ Orionis cluster whose orbital parameters have been derived $(P=8.72 \pm 0.02 \mathrm{~d}$, Sacco et al. (2008) also derived a later spectral type, K9.5, and found lithium in both spectra).

Our observations confirm and contradict some of the observational results above. First, using the same method as for Mayrit 797272, we derived an M3 \pm 1 spectral type for Mayrit 102101AB, which matches more closely the spectro-photometric cluster sequence than previous determinations. The $\mathrm{H} \alpha$ emission in our ALFOSC spectrum $(E W(\mathrm{H} \alpha)=-$ $32 \pm 2 \AA$ ) is not as strong as measured by Wolk (1996), although it still satisfyies the accreting empirical criterion of Barrado $y$ Navascués \& Martín (2003) (the White \& Basri 2003's criterion cannot be applied because of our poor wavelength resolution). The strong (variable?) $\mathrm{H} \alpha$ emission might originate from material interchange between the two binary components or accretion from a disc. As already noticed by Hernández et al. (2007), the IRAC [3.8] - [8.0] colour of Mayrit 102101AB is only 0.27 $\pm 0.05 \mathrm{mag}$. Furthermore, the apparent flux excess at the MIPS [24] passband is due to an overdensity in a large intracluster dust cloud, and not to the star itself (Sect. A). This overdensity may also be responsible for the slight excess at [8.0]. The close separation between Mayrit 102101A and $\mathrm{B}$, of $\left(a_{1}+a_{2}\right) \sin i=11.6$ $\pm 0.3 R_{\odot}$ (i.e. a few objects radii ${ }^{3}$, Sacco et al. 2008), prevents the formation of inner disc(s) surrounding one (or both) component(s). From our point of view, the origin of the X-ray and $\mathrm{H} \alpha$ emissions is explained more accurately by the mutual interaction of the two binary components rather than by the presence of an "evolved disc". In any case, we do not completely discard the presence of a cool, circum-binary disc at several tens of solar radii.

\subsubsection{Mayrit 264077}

Mayrit 264077 (S Ori J053902.1-023501) is an object at the stellar/substellar boundary in $\sigma$ Orionis, with a most probable mass of $0.061 M_{\odot}$ (Caballero et al. 2004, 2007). It had, by far, the reddest near-infrared colours among the objects in the Caballero et al. (2007)'s survey $\left(J-K_{\mathrm{s}}=1.84 \pm 0.05 \mathrm{mag}\right.$ [2MASS], [3.6] $-[8.0]=1.70 \pm 0.06 \mathrm{mag}$ ) and was in a differentiated location in the [3.6] $-[8.0]$ versus $J-K_{\mathrm{s}}$ colour-colour diagram. Hernández et al. (2007) also measured [24] $=6.45 \pm 0.03$ mag (consistent with our photometry) and identified Mayrit 264077 to be a class II object. It has no spectroscopic information.

The spectral energy distribution in Fig. 4 complements that shown in Caballero et al. (2007). The presence of a circumstellar disc is obvious in the photometric data; the ALFOSC spectrum powerfully reinforces the disc's existence. We determined an M3 \pm 1 spectral type for Mayrit 264077, identical to that of Mayrit 102101AB. However, the $\mathrm{H} \alpha$ emission was stronger: $E W(\mathrm{H} \alpha)=-72 \pm 4 \AA$. We also detected the lines of [O I] $\lambda 6300.3 \AA$ and [S II] $\lambda \lambda 6716.4,6730.8 \AA$ in forbidden emission (the [N II] $\lambda 6583.4 \AA$ is blended with the strong $\mathrm{H} \alpha$ line).

We note that the I-band light curve of Mayrit 264077 displayed short-, mid-, and long-scale variations in the original data of Caballero et al. (2004). Since it was one of the brightest targets in their sample and its photometry was, therefore, close to the non-linear limit of the detector, Mayrit 264077 was discarded conservatively from the detailed analysis. However, returning to the original light curve, it is quite possible that those variations were intrinsic to the object and not due to seeing variations and non-linear effects. As shown by Caballero et al. (2006), T Taurianalog brown dwarfs in $\sigma$ Orionis with strong $\mathrm{H} \alpha$ emission, forbidden lines, and near-infrared flux excess also display large amplitude photometric variations on all considered timescales.

Mayrit 264077 might be a low-mass T Tauri star with a high extinction bluewards of $\sim 1.5 \mu \mathrm{m}$ accompanied by a flux excess redwards of this wavelength (i.e. the central object is a star brighter than $J=14.44 \pm 0.04 \mathrm{mag}$, and not a brown dwarf).

\footnotetext{
3 The objects are still contracting and have radii much larger than measured for field dwarfs of the same spectral type.
} 


\subsubsection{Mayrit 358154}

Mayrit 358154 (S Ori J053855.4-024121) was another class II, brown dwarf candidate without spectroscopy in the works of Caballero et al. (2007) and Hernández et al. (2007). It was not detected during the optical/near-infrared survey in Caballero et al. (2004) because it was in the glare of the $3.8 \mathrm{mag}-V$ multiple star $\sigma$ Ori. Since Mayrit 358154 is 1.2 mag fainter than Mayrit 264077, we expect that the first object is a bona fide brown dwarf, even accounting for a possible edge-on disc. To date, there is only one $\sigma$ Orionis star fainter than $J \sim 14.5 \mathrm{mag}$, and it is the source of a well known Herbig-Haro object (HH 446 [Mayrit 633105] - Reipurth et al. 1998; Andrews et al. 2004). However, Mayrit 358154 is even fainter in $J$ than this HerbigHaro star. Mayrit 358154 is at $8.8 \operatorname{arcsec}$ to the north of a brighter photometric, cluster-member, candidate from Sherry et al. (2004). The hypothetical primary was not, however, in the comprehensive cluster catalogue of Caballero (2008c).

The ALFOSC spectrum and the spectral energy distribution strenghten the evidence that Mayrit 358154 has a circum(sub)stellar disc. The flux excess in the [8.0] passband is clearly evident, although the spectral energy distribution appears to decline sharply again at redder wavelengths (our upper limit to the [24] passband is quite restrictive). The distribution has a peculiar feature in the $H$ band (Mayrit 633105 should be brighter at $1.6 \mu \mathrm{m}$ ), whose true origin we fail to ascertain; the feature cannot be explained by photometric error. The $\mathrm{H} \alpha$ emission is even stronger than in previous objects $(E W(\mathrm{H} \alpha)=-190 \pm 20 \AA)$ and we could identify the [O I], [S II], and [N II] forbidden emission lines in the studied wavelength interval. The derived spectral type, M5 \pm 1 , is consistent with Mayrit 358154 being a high-mass brown dwarf in $\sigma$ Orionis (the earliest brown dwarf candidates known to date in the cluster have M5.5 spectral types). Although its properties are in the substellar domain, Mayrit 358154 displays most of the phenomena observed in classical T Tauri stars.

\subsection{Background galaxies}

UCM0537-0227 was the faintest new $\sigma$ Orionis photometric member candidate in the Mayrit catalogue of Caballero (2008c). He indicated the DENIS $i$ and 2MASS $J H K_{\mathrm{S}}$ magnitudes for the object, from which one could derive a mass in the middle of the brown dwarf domain, if it belonged to the cluster. He also noticed that UCM0537-0227 possibly had an extended, non-stellar point spread function. Its extremely red 2MASS $\left(J-K_{\mathrm{s}}=2.34 \pm\right.$ $0.11 \mathrm{mag}$ ) and IRAC/Spitzer colours measured by Caballero et al. (2008) encouraged us to obtain follow-up spectroscopy. The low-resolution optical spectrum (top panel of Fig. 3) and the spectral energy distribution of UCM0537-0227 correspond to an emission-line, star-forming galaxy at a spectroscopic redshift $z_{\text {sp }}=0.1009 \pm 0.0002$ with strong PAH features and a moderate star formation rate.

UCM0536-0239 ([HHM2007] 668) and its odd spectral energy distribution from the $I$ band to [8.0] was first identified by Caballero (2006). Just afterwards, Hernández et al. (2007) measured its [24]-band magnitude and presented it as one of the few class I object candidates in $\sigma$ Orionis. Caballero (2007b) noticed that UCM0536-0239 (incorrectly named "Mayrit 111208" in his work) had quite blue $I-J$ and extremely red $J-K_{\mathrm{s}}$ colours for its faint magnitude $(J=16.14 \pm 0.10 \mathrm{mag})$ and found that it was an X-ray emitter detected with the ACIS and HRC-I instruments onboard Chandra. The (sub)stellar nature of UCM05360239 was placed in doubt by Caballero (2008c), who measured a possible extended point spread function in public optical images. Finally, Skinner et al. (2008) measured the mean photon energy of UCM0536-0239 at $\bar{E}=3.40 \mathrm{keV}$ from ACIS/Chandra data, which is quite a high value for a normal star, but typical of active galactic nuclei. The ALFOSC spectrum (bottom panel of Fig. 3) and the spectral energy distribution of UCM0536-0239 favour its classification as a Type 1 obscured quasi-stellar object at $z_{\mathrm{sp}}=0.2362 \pm 0.0005$.

\section{Summary}

We have obtained, compiled, and analysed low-resolution spectroscopy $(R \sim 500)$, acquired with ALFOSC at the Nordic Optical Telescope, and spectral energy distributions, from the optical $V$ band to the mid-infrared MIPS/Spitzer [24] band, of nine selected sources towards the young $\sigma$ Orionis cluster. These sources cover a wide range in brightness, which was expected to translate into a wide range in mass and spectral type. Of the nine optical spectra presented, six correspond to objects without previous spectroscopic observations.

Two of the targets in our sample were identified as low redshift $(z \approx 0.10-0.24)$ galaxies with prominent emissionlines in the optical and high fluxes at $8-24 \mu \mathrm{m}$ due to an active galactic nucleus (UCM0536-0239) and a burst of star formation (UCM0537-0227). Another target seemed to be a solarlike star in the field. Another five objects were classified as bona-fide cluster members with spectral types in the interval A2-6 to M4-6. We failed to detect spectroscopic indicators of youth in the remaining solar-like, cluster member candidate (Mayrit 968292).

Four of the five confirmed $\sigma$ Orionis members display peculiar features in their optical spectra and energy distributions:

- Mayrit 459340 is an A2-6-type Herbig Ae/Be star with strong $\mathrm{H} \alpha$ emission $(E W(\mathrm{H} \alpha)=-10 \pm 1 \AA)$, flux excess at the MIPS/Spitzer [24] band, and a probable scattering edgeon disc, in analogy to UX Ori.

- Mayrit 102101AB is a young, low-mass, spectroscopic binary whose $\mathrm{H} \alpha$ emission could be variable and have originated from the mass transfer between A and B.

- Mayrit 264077 is an accretor at the substellar boundary with a developed disc and a flux excess that extends longwards of $24 \mu \mathrm{m}$.

- Mayrit 358154 remains as the second faintest $\sigma$ Orionis member with spectroscopic features of youth and DENIS and 2MASS identification. Because of its strong, probably broad, $\mathrm{H} \alpha$ emission (of up to about $-200 \AA$ ), [O I], [S II] , and $[\mathrm{N}$ II] forbidden lines in emission, M5 \pm 1 spectral type, flux excess at the four IRAC/Spitzer channels, and faintness $(i=$ $18.1 \pm 0.2 \mathrm{mag}$ ), we have classified it as a "classical T Tauri brown dwarf".

The fifth cluster member, Mayrit 797272, is an X-ray flaring star without a disc. We have also presented three previously unknown mid-infrared sources without optical/near-infrared counterparts. The $\sigma$ Orionis cluster still conceals many astonishing surprises; simple low-resolution spectroscopy and analysis of spectral energy distributions are suitable tools for finding them.

\section{Appendix A: The new infrared sources $\sigma$ Ori IRS2, $3 A$, and $3 B$}

Dust clouds exist at relatively short separations from the Trapezium-like system $\sigma$ Ori AF-B. First, 
van Loon \& Oliveira (2003) discovered $\sigma$ Ori IRS1, a mid-infrared source at just $\sim 3$ arcsec to the OB triple stars, which they associated with a dust cloud. This source originated in the photoevaporation of the outer layers of a binary low-mass star that lies on its densest part (Sanz-Forcada et al. 2004; Caballero 2005, 2007a,b; Turner et al. 2008; Skinner et al. 2008; Bouy et al. 2008). Next, Caballero et al. (2008) discovered a "cloud or shell of cool gas surrounding [...] $\sigma$ Ori". Although the cloud is obvious in the MIPS/Spitzer [24]-band image (right window in Fig. 1; see also Fig. 2 in Hernández et al. 2007), it has received no attention in the literature.

The brightest, densest knot in the cloud falls at a couple of arcseconds from our spectroscopic binary Mayrit 102101AB, whose [24]-band photometry could not be extracted properly because of the knot. We refer to this source as $\sigma$ Ori IRS2, following the nomenclature introduced by van Loon \& Oliveira (2003) for unidentified mid-infrared sources without (known) optical/near-infrared counterpart. There are another two additional mid-infrared sources associated with a longitudinal arrangement in the cloud, to the northeast of $\sigma$ Ori AF-B. Since they appeared to form part of the same structure, we called them $\sigma$ Ori IRS3A and 3B.

The approximate central coordinates (J2000) of the new infrared sources are $05 \quad 38 \quad 51.0 \quad-02 \quad 3624$ ( $\sigma$ Ori IRS2), $053847.6-023514$ ( $\sigma$ Ori IRS3A), and $053858.7-023530$ ( $\sigma$ Ori IRS3B). The origin of the dust cloud that harbours $\sigma$ Ori IRS2, 3A, and 3B is as yet unknown.

Acknowledgements. We thank the anonymous referee, G. Barro Calvo, M. Rego, V. Villar, and J. Zamorano for helpful comments. The data presented here have been taken using ALFOSC, which is owned by the Instituto de Astrofísica de Andalucía (IAA) and operated at the Nordic Optical Telescope under agreement between IAA and the NBIfAFG of the Astronomical Observatory of Copenhagen. Partial financial support was provided by the Universidad Complutense de Madrid, the Spanish Virtual Observatory, the Spanish Ministerio Educación y Ciencia, and the European Social Fund under grants AyA200502750, AyA2005-04286, AyA2005-24102-E, and AyA2007-67458 of the Programa Nacional de Astronomía y Astrofísica and by the Comunidad Autónoma de Madrid under PRICIT project S-0505/ESP-0237 (AstroCAM).

\section{References}

Andrews, S. M., Reipurth, Bo, Bally, J., \& Heathcote, S. R. 2004, ApJ, 606, 353 Bahcall, J. N., \& Soneira, R. M. 1980, ApJS, 44, 73

Barrado y Navascués, D., \& Martín, E. L. 2003, AJ, 126, 2997

Barrado y Navascués, D., Zapatero Osorio, M. R., Béjar, V. J. S., et al. 2001, A\&A, 377, L9

Béjar, V. J. S., Zapatero Osorio, M. R., \& Rebolo, R. 1999, ApJ, 521, 671

Béjar, V. J. S., Martín, E. L., Zapatero Osorio, M. R., et al. 2001, ApJ, 556, 830

Béjar, V. J. S., Zapatero Osorio, M. R., \& Rebolo, R. 2004, AN, 325, 705

Bibo, E. A., \& Thé, P. S. 1991, A\&AS, 89, 319

Bouy, H., Huélamo, N., Martín, E. L., et al. 2008, A\&A, in press,

[arXiv:0808.3890]

Caballero, J. A. 2005, AN, 326, 1007

Caballero, J. A. 2006, Ph.D. Thesis, Universidad de La Laguna, Spain

Caballero, J. A. 2007a, A\&A, 466, 917

Caballero, J. A. 2007b, AN, 328, 917

Caballero, J. A. 2008a, MNRAS, 383, 375

Caballero, J. A. 2008b, MNRAS, 383, 750

Caballero, J. A. 2008c, A\&A, 478, 667

Caballero, J. A., Béjar, V. J. S., Rebolo, R., \& Zapatero Osorio, M. R. 2004, A\&A, 424, 857

Caballero, J. A., Martín, E. L., Zapatero Osorio, M. R., et al. 2006, A\&A, 445, 143

Caballero, J. A., Béjar, V. J. S., Rebolo, R., et al. 2007, A\&A, 470, 903

Caballero, J. A., Barrado y Navascués, D., Béjar, V. J. S., et al. 2008, 14th Cambridge Workshop on Cool Stars, Stellar Systems, and the Sun ASP Conf. Ser., 384, Proceedings of the conference held 5-10 November, 2006, at the Spitzer Science Center and Michelson Science Center, Pasadena, California, USA, ed. G. van Belle, Poster \#31 (in DVD).
Danks, A. C., \& Dennefeld, M. 1994, PASP, 106, 382

Downes, R. A., \& Keyes, C. D. 1988, AJ, 96, 777

Engelbracht, C. W., Blaylock, M., Su, K. Y. L., et al. 2007 PASP, 119, 994

Epchtein, N., de Batz, B., Capoani, L., et al. 1997, Msngr, 87, 27

European Space Agency (ESA), The Hipparcos and Tycho catalogues, ESA SP1200, 1997

Feigelson, E. D., Broos, P., Gaffney, J. A., III, et al. 2002 ApJ, 574, 258

Franciosini, E., Pallavicini, R., \& Sanz-Forcada, J. 2006 A\&A, 446, 501

Gatti, T., Natta, A., Randich, S., Testi, L., \& Sacco, G. 2008, A\&A, 481, 423

González-García, B. M., Zapatero Osorio, M. R., Béjar, V. J. S., et al. 2006, A\&A, 460, 799

Güdel, M., Schmitt, J. H. M. M., Benz, A. O., \& Elias, N. M., II 1995, A\&A, 301,201

Hernández, J., Hartmann, L., Megeath, T., et al. 2007, ApJ, 662, 1067

Høg, E., Fabricius, C., Makarov, V. V., et al. 2000, A\&A, 355, L27

Jacoby, G. H., Hunter, D. A., \& Christian, C. A. 1984, ApJS, 56, 257

Kenyon, S. J., \& Hartmann, L. 1995, ApJS, 101, 117

Kenyon, M. J., Jeffries, R. D., Naylor, T., Oliveira, J. M., \& Maxted, P. F. L. 2005, MNRAS, 356, 89

Lee, T. A. 1968, ApJ, 152, 913

Leggett, S. K., Allard, F., Geballe, T. R., Hauschildt, P. H., \& Schweitzer, A. 2001, ApJ, 548, 908

van Loon, J. Th., \& Oliveira, J. M. 2003, A\&A, 405, L33

López-Santiago, J., \& Caballero J. A. 2008, A\&A, in press, doi: 10.1051/0004-6361:200810475

Luhman, K. L., Hernández J., Downes, J. J., Hartmann, L., \& Briceño, C. 2008, ApJ, in press, [arXiv: 0808.0471]

Martín, E. L., \& Kun, M. 1996, A\&AS, 116, 467

Martín, E. L., Rebolo, R., \& Zapatero Osorio, M. R. 1996, ApJ, 469, 706

Martín, E. L., Zapatero Osorio, M. R., Barrado y Navascués, D., Béjar, V. J. S., \& Rebolo, R. 2001a, ApJ, 558, L117

Martín, E. L., Dougados, C., Magnier, E., et al. 2001b, ApJ, 561, L195

Maxted, P. F. L., Jeffries, R. D., Oliveira, J. M., Naylor, T., \& Jackson, R. J. 2008, MNRAS, 385, 2210

Mayne, N. J., \& Naylor, T. 2008, MNRAS, 386, 261

Monet, D. G., Levine, S. E., Canzian, B., et al. 2003, AJ, 125, 984

Montes, D., Martín, E. L., Fernández-Figueroa, M. J., Cornide, M., \& de Castro, E. 1997, A\&AS, 123, 473

Oliveira, J. M., Jeffries, R. D., van Loon, J. Th., \& Rushton, M. T. 2006, MNRAS, 369, 272

Pojmański, G. 2002, AcA, 52, 397

Reipurth, Bo, Bally, J., Fesen, R. A., \& Devine, D. 1998 Nature, 396, 343

Robrade, J., \& Schmitt, J. H. M. M. 2005, A\&A, 435, 1073

ROSAT Consortium 2000, VizieR On-line Data Catalog: IX/30, originally published in: ROSAT News 72, 25-May-2000

Sacco, G. G., Franciosini, E., Randich, S., \& Pallavicini, R. 2008, A\&A, 488, 167

Sanz-Forcada, J., Franciosini, E., \& Pallavicini, R. 2004, A\&A, 421, 715

Scholz, A., \& Eislöffel, J. 2004 A\&A, 419, 249

Scholz, A., \& Jayawardhana, R. 2008 ApJ, 672, L49

Sherry, W. H., Walter, F. M., \& Wolk, S. J. 2004, AJ, 128, 2316

Sherry, W. H., Walter, F. M., Wolk, S. J., \& Adams, N. R. 2008, AJ, 135, 1616

Skinner, S. L., Sokal, K. R., Cohen, D. H., et al. 2008, ApJ, 683, 796

Skrutskie, M. F., Cutri, R. M., Stiening, R., et al. 2006, AJ, 131, 1163

Stelzer, B., Micela, G., \& Neuhäuser, R. 2004, A\&A, 423, 1029

Stephenson, C. B. 1986, ApJ, 300, 779

Thé, P. S., de Winter, D., \& Pérez, M. R. 1994, A\&AS, 104, 315

Turner, N. H., ten Brummelaar, T. A., Roberts, L. C., et al. 2008, AJ, 136, 554

Valdes, F., Gupta, R., Rose, J. A., Singh, H. P., \& Bell, D. J. 2004, ApJS, 152, 251

White, N. E., Giommi, P., \& Angelini, L. 2000, VizieR On-line Data Catalog: IX/31, originally published in: Laboratory for High Energy Astrophysics (LHEA/NASA), Greenbelt (2000)

White, R. J., \& Basri, G. 2003, ApJ, 582, 1109

Wolk, S. J. 1996, Ph.D. Thesis, State University New York at Stony Brook, USA Zamorano, J., Rego, M., Gallego, J. G., et al. 1994, ApJS, 95, 387

Zapatero Osorio, M. R., Béjar, V. J. S., Martín, E. L., et al. 2000, Science, 290, 103

Zapatero Osorio, M. R., Béjar, V. J. S., Pavlenko, Ya., et al. 2002a, A\&A, 384, 937

Zapatero Osorio, M. R., Béjar, V. J. S., Martín, E. L., Barrado y Navascués, D., \& Rebolo, R. 2002b, ApJ, 569, L99

Zapatero Osorio, M. R., Béjar, V. J. S., Martín, E. L., et al. 2002c, ApJ, 578, 536 Zapatero Osorio, M. R., Caballero, J. A., Béjar, V. J. S., et al. 2007, A\&A, 472, L9

Zinnecker, H., \& Yorke, H. W. 2007, ARA\&A, 45, 481 